\title{
Effects of Interpolation on the Inverse Problem of Electrocardiography
}

\author{
Y S Dogrusoz ${ }^{1}$, L R Bear ${ }^{2}$, J Bergquist ${ }^{3}$, R Dubois ${ }^{2}$, W Good ${ }^{3}$, R S MacLeod ${ }^{3}$, A Rababah $^{4}$, J Stoks ${ }^{5}$ \\ ${ }^{1}$ Electrical and Electronics Engineering Department, METU, Ankara, Turkey \\ ${ }^{2}$ IHU-LIRYC, Université de Bordeaux, Bordeaux, France \\ ${ }^{3}$ Dept of Biomedical Engineering and SCI Institute, University of Utah, Salt Lake City (UT), USA \\ ${ }^{4}$ Faculty of Computing, Engineering and the Built Environment, Ulster University, United Kingdom \\ ${ }^{5}$ Maastricht University, Maastricht, The Netherlands
}

\begin{abstract}
Electrocardiographic Imaging (ECGI) aims to reconstruct electrograms from the body surface potential measurements. Bad leads are usually excluded from the inverse problem solution. Alternatively, interpolation can be applied. This study explores how sensitive ECGI is to different bad-lead configurations and interpolation methods. Experimental data from a Langendorff-perfused pig heart suspended in a human-shaped torso-tank was used. Six different bad lead cases were designed based on clinical experience. Inverse problem was solved by applying Tikhonov regularization i) using the complete data, ii) badleads-removed data, and iii) interpolated data, with 5 different methods. Our results showed that ECGI accuracy of an interpolation method highly depends on the location of the bad leads. If they are in the high-potential-gradient regions of the torso, a highly accurate interpolation method is needed to achieve an ECGI accuracy close to using complete data. If the BSP reconstruction of the interpolation method is poor in these regions, the reconstructed electrograms also have lower accuracy, suggesting that bad leads should be removed instead of interpolated. The inverseforward method was found to be the best among all interpolation methods applied in this study in terms of both missing BSP lead reconstruction and ECGI accuracy, even for the bad leads located over the chest.
\end{abstract}

\section{Introduction}

In electrocardiographic imaging (ECGI), the aim is to reconstruct high-resolution images of cardiac electrical activity from body surface potential (BSP) measurements [1]. It is a promising tool for clinical applications such as proposing regions of abnormal atrial or ventricular activity before ablation procedures, providing information on regions with ischemic or infarcted tissue, and revealing conduction abnormalities [1]. Multi-electrode signal ac- quisition systems are used with the number of electrodes ranging from 32 to 219 in ECGI [2]. However, due to broken electrodes or simultaneously attaching other electrodes such as defibrillation patches or CARTO leads on the body surface, some ECG leads cannot be observed. These leads are either excluded from the inverse reconstructions, or interpolated based on remaining leads [3-5]. In their work, Bear et al. compared the ECGI performance for removing as opposed to reconstructing the missing leads using linear interpolation [5]. The aim in this collaborative study is to extend the work in [5], and compare the performances of different interpolation techniques for 1) reconstructing the missing ECG leads, 2) solving the inverse ECG problem, for a variety of bad lead configurations.

Six different bad lead cases were designed based on clinical experience, and bad leads were interpolated using 5 different interpolation methods. The inverse problem was solved by applying Tikhonov regularization using the complete data, bad leads removed data and interpolated data. Results were evaluated qualitatively and quantitatively in terms of interpolated ECG signals, reconstructed electrograms and the corresponding activation time maps.

\section{Methods}

Experimental data came from a Langendorff-perfused pig heart suspended in a human-shaped torso-tank. Epicardial electrograms were recorded during RV pacing using a 108-electrode array, simultaneously with ECGs from 128 electrodes embedded in the tank surface [6]. Data consisted of 30 seconds of ECG recordings, with 31 beats. Preprocessing was applied to the ECG signals to filter the high frequency noise, $50 \mathrm{~Hz}$ line interference and baseline drift noise.

Six different bad lead cases were designed based on clinical experience: (BL1) Defibrillation patches - 1, (BL2) Defibrillation patches - 2, (BL3) CARTO patches, on the front and the back of torso, (BL4) Shoulders missing, (BL5) Sides of the torso missing, and (BL6) Combination 
of BL2-BL4, to represent a worst case scenario. In addition, one of the torso leads, which recorded a poor quality signal, was included in all bad lead configurations.

Five different interpolation methods were used to reconstruct the missing leads: 1) Laplacian interpolation (LAP) (SCIRun: http://www.sci.utah.edu/cibcsoftware/scirun.html), 2) Inverse-distance square weighting (IDS) [7], 3) Kriging method (KRI) [8], 4) The inverseforward method (I-F) [3], 5) A hybrid method [9]. In the training step of this hybrid method, first only the first beat (HYB1), then the first 10 beats (HYB2), were used.

The forward problem relating epicardial data to BSPMs was solved using the boundary element method (BEM) [10] assuming a homogeneous volume between the heart and torso surfaces. The inverse problem was solved using Tikhonov regularization [11] for each beat in the signal ( 31 beats). We obtained solutions by first using complete leads (Full), by removing bad leads (NoInt), and finally by interpolating bad leads (LAP, IDS, KRI, I-F, HYB1 and HYB2).

\subsection{Results}

\section{Evaluation of Interpolated BSPs}

For each bad lead configuration, interpolated signals were compared to the true recordings (except the one lead with poor quality signal), in terms of correlation coefficient (CC) and absolute-amplitude-difference.

$\mathrm{CC}$ values were obtained between the measured and interpolated signals for each bad-lead configuration during the QRS interval. Their median values were then calculated for each beat. For almost all interpolation methods and bad-lead configurations, we obtained mean $\mathrm{CC}$ values of 0.98-1.00, with the exception of the IDS method, which had CC values of 0.93 and 0.97 for BL1 and BL2 configurations, respectively, indicating the morphologies of the recorded signals are well-captured in the interpolated signals.

Next, absolute differences of peak-to-peak signal amplitudes were calculated between the measured and interpolated signals for each bad-lead configuration during the QRS interval. The mean values of the median absoluteamplitude-differences over all beats are presented in Figure 1. For the BL1 configuration, the highest values were obtained for the Laplacian and the Hybrid approaches, with a relative error (RE) of approximately $20 \%$, followed by IDS, which had a RE of $15.88 \%$. KRI and I-F methods had the lowest amplitude differences, with RE values of $6.56 \%$ and $5.81 \%$, respectively. For BL2, Laplacian and the Hybrid methods had RE values reduced to approximately $5 \%$ similar to that of KRI. RE value of the I-F method was the smallest, with a value of $3.96 \%$. On the other hand, the RE value for the IDS method remained high $(16.69 \%)$. For the BL3 configuration, for all inter-

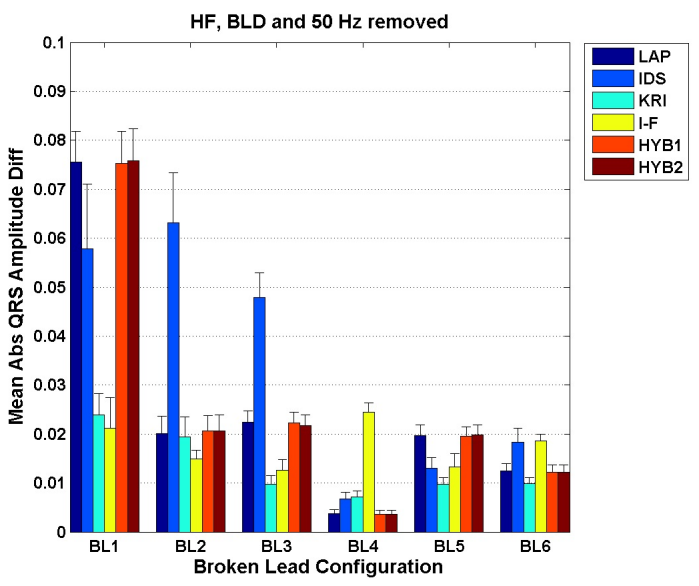

Figure 1. The mean values of the median absoluteamplitude-differences for all bad-lead configurations and interpolation methods.

polation methods except the IDS, amplitude differences were small, with RE values less than $7 \%$, and as low as $2.86 \%$ for the KRI method. IDS, on the other hand, had the largest amplitude difference with a RE of $14.10 \%$. For BL4, all methods had RE values less than $2 \%$, except the I-F method, which yielded a RE of $6.95 \%$. For BL5, and BL6, all RE values were below $10 \%$ and $5 \%$, respectively. These differences in the amplitudes indicate that the interpolation methods may alter the amplitudes of the signals, despite their high performances in capturing the signal morphologies.

In figure 2, we show the frontal view of the body surface potential maps of the recorded and interpolated signals, for the BL3 configuration. The bad-leads were marked as white dots on the torso surface. In this bad-lead configuration, the missing leads were located close to the heart, in a region of high-potential-gradient. The IDS method failed to capture the local minimum on the torso surface, yielding high signal amplitude errors. Laplacian, Hybrid and KRI methods had better fidelity to the true distributions than the IDS, but the details around the global minimum region were best represented by the I-F method. These maps are in agreement with the absolute-amplitude-differences and the corresponding RE values presented earlier. We had similar observations for the BSP maps for BL6.

\section{Evaluation of Reconstructed EGMs}

The reconstructed electrograms (EGM) were evaluated in terms of CC values with two different "gold-standard" definitions. First, all reconstructions obtained by including all measured leads and by removing/interpolating bad leads were compared with the true sock recordings. Then, the reconstructions with the complete-lead recordings were assigned as the "gold standard" and EGMs ob- 

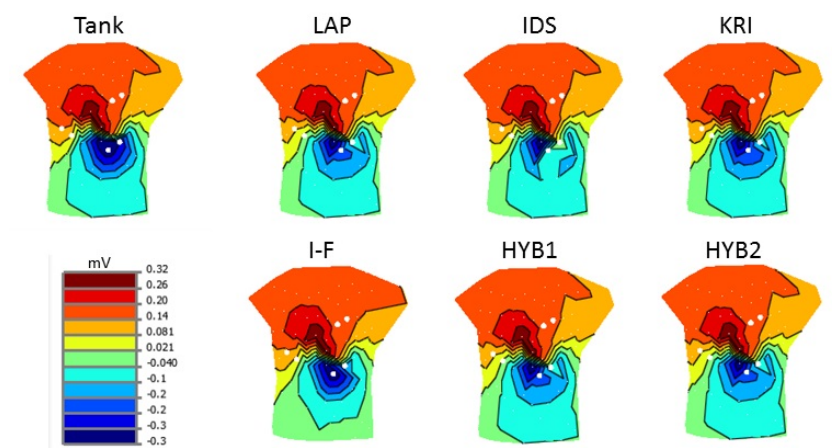

Figure 2. Sample body surface potential maps for the bad lead configuration, BL3. Bad leads were marked as white dots on the torso surface. Top left panel: true BSP recordings (ground truth); Remaining maps: Interpolated BSPs, with labels on the top indicating the interpolation method.

tained by removing/interpolating bad leads were compared to those reconstructions.

Figure 3 shows the mean CC values when the "gold standard" are the measured sock potentials. In BL1 and BL2, all methods had mean CC values of $0.60-0.67$, with the lowest $\mathrm{CC}$ of 0.6 corresponding to removing bad leads in BL1, and IDS in BL2. In BL3, we observed a significant drop in the performance of IDS, with mean CC of 0.39, which was followed by KRI, with mean CC of 0.50 . I-F method yielded a result comparable to using the complete leadset, or removing broken leads, with a CC value of 0.63. In BL4, all methods had similar performances, but the I-F method had slightly larger mean CC values than the other reconstructions. In BL5, all methods except the IDS performed similarly, with mean CC values of 0.62-0.66. IDS had the lowest mean CC of 0.59. BL6 results were similar to the BL3 results, which is not surprising, since BL3 is a subset of BL6. Again, the mean CC values of the IDS method were the lowest of all reconstructions.

Figure 4 shows the mean CC values to compare removing versus interpolating bad leads, with the complete-lead solutions as the "gold standard". In BL1 and BL2, all interpolation methods had mean $\mathrm{CC}$ values ranging between 0.96-0.99, where removing bad leads yielded mean CC of 0.99-1.00. In BL4 and BL5, where the bad leads are located on the shoulders, and on the sides of the torso respectively, there was no difference between the reconstructions with removing or interpolating bad leads, all yielding mean CC of 1.00. BL3 and BL6, on the other hand, which have bad leads near the heart over the high-potential-gradient region of the torso, displayed differences between the interpolation methods. In both configurations, removing bad leads resulted in mean $\mathrm{CC}$ of 0.99 , whereas the interpolated reconstructions had lower values. In BL3, the high-

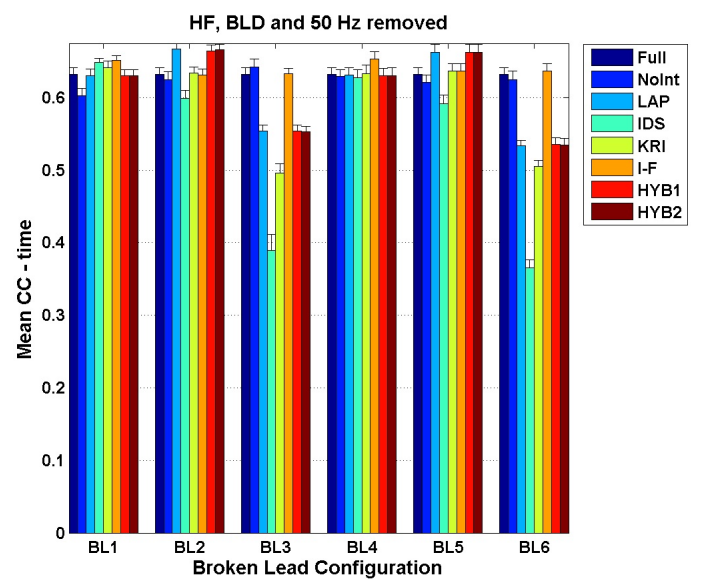

Figure 3. Mean CC values for all electrogram reconstructions when the "gold standard" are the measured sock potentials.

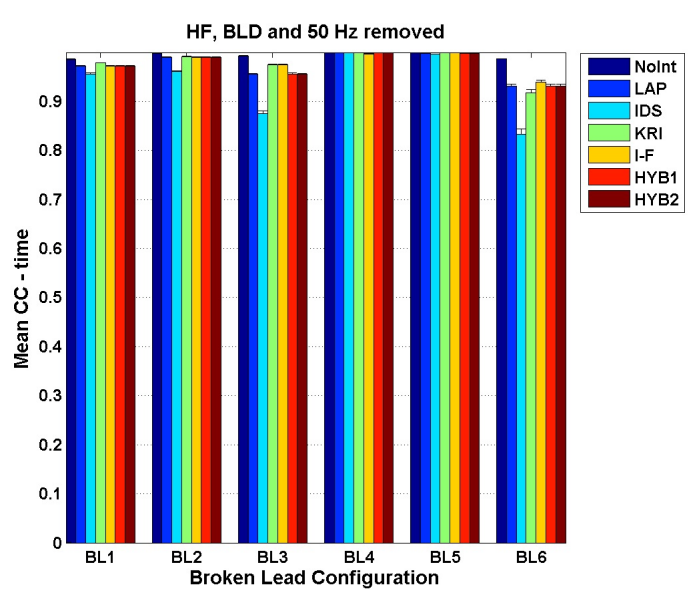

Figure 4. Mean CC values for the electrogram reconstructions by removing or interpolating the missing leads, when the "gold standard" are the reconstructions using the complete leadset.

est two mean CC values correspond to KRI (0.98) and I-F (0.97), followed by LAP, HYB1 and HYB2 (0.96). In BL6, the gaps between the $\mathrm{CC}$ values were higher, with mean $\mathrm{CC}$ values of 0.92-0.94 for all methods except the IDS. For both bad lead configurations BL3 and BL6, IDS had the worst performance, with mean $\mathrm{CC}$ of 0.88 and 0.83 , respectively.

\section{Evaluation of activation times}

Activation times (AT) were computed by the method proposed in [12] for all inverse solutions, and compared with the ATs obtained from the sock measurements using Pearson's CC. Localization errors (LE) for the pac- 
ing site were also calculated for all solutions. In BL3 and BL4, where there are missing leads on the high-potentialgradient torso regions, all methods had lower $\mathrm{CC}$ values compared to removing bad leads. The drop in $\mathrm{CC}$ values were within a range of 3.83-8.19\% in BL3, and 1.65-6.51 $\%$ in BL6, except for the IDS method. IDS was the worst of all interpolation methods with $\mathrm{CC}$ value decreasing by $20 \%$ in BL3 and $17 \%$ in BL6. I-F and HYB1 had the smallest LE values for BL3 and BL6, respectively, with values approximately $12 \%$ lower than removing bad leads. IDS performed the worst among all methods, especially in BL3 and BL6, where \% increase in the LE values compared to removing bad leads was as high as $34 \%$.

\section{Discussion and Conclusion}

Our results show that the effects of interpolation on ECGI depends on the interpolation accuracy over the highpotential-gradient regions of the torso surface, which usually lie on the torso front. If the features of the BSP maps are well-reconstructed at the missing leads by the applied interpolation method, this in turn results in EGM reconstructions similar to those obtained by using complete leads. Otherwise, interpolation deteriorates the EGM reconstructions. This is mainly observed in BL3, where some of the missing electrodes are located directly over the heart surface, and BL6, which includes BL3. IDS method yielded BSP reconstructions with the largest amplitude differences in these regions, which in turn negatively effected its ECGI performance. I-F method, which had the best performance for reconstructing the BSP maps over these high-potential-gradient regions also performed very well in terms of ECGI accuracy. If the bad leads do not coincide with the high-potential-gradient regions of the torso, as in BL4 (bad leads on the shoulders) all interpolation methods performed equally well, and similar to using complete leads, and removing the bad leads. These results are in agreement with the observations of [5]. Even though we observed a decrease in the AT-CC values of the interpolation methods compared to removing bad leads, pacing site localization accuracy improved with interpolation, with IF and HYB1 outperforming the other methods.

Interpolation of bad leads yields EGM reconstructions as good as removing the bad leads, if the interpolation accuracy is high in the high-potential-gradient regions of the torso. If this accuracy cannot be guaranteed, bad leads should be removed instead of interpolated. I-F method performs the best among all interpolation methods applied in this study.

\section{Acknowledgements}

This is a collaborative work for the Consortium on ECG Imaging (CEI); all authors contributed equally. It was sup- ported by the French National Research Agency (ANR10-IAHU04-LIRYC), Nora Eccles Treadwell Foundation for Cardiovascular Research, and the National Institute of General Medical Sciences of the National Institutes of Health under grant number P41 GM103545-18, and the European Union's INTERREG VA Programme, managed by the Special EU Programmes Body (SEUPB).

\section{References}

[1] Cluitmans MJM, et al. Noninvasive reconstruction of cardiac electrical activity: update on current methods, applications and challenges. Neth Heart J 2015;23:301-311.

[2] Hoekema R, Uijen GJH, van Oosterom A. On selecting a body surface mapping procedure. Journal of Electrocardiology 1999;32(2):93-101.

[3] Burnes JE, et al. A field-compatible method for interpolating biopotentials. Ann Biomed Eng 1998;26(1):37-47.

[4] Sapp JL, Dawoud F, Clements JC, Horáček BM. Inverse solution mapping of epicardial potentials: quantitative comparison with epicardial contact mapping. Circ Arrhythm Electrophysiol 2012;5(5):1001-1009.

[5] Bear L, et al. Accuracy of lead removal vs linear interpolation in non-invasive electrocardiographic imaging (ECGI). In Computing in Cardiology Conference (CinC). 2015; 941-944.

[6] Bear L, et al. Effects of ecg signal processing on the inverse problem of electrocardiography. In Computing in Cardiology Conference (CinC). 2018;

[7] Shepard D. A two-dimensional interpolation function for irregularly-spaced data. In Proceedings of the 1968 23rd ACM national conference. ACM, 1968; 517-524.

[8] van Beers WCM, Kleijnen JPC. Kriging for interpolation in random simulation. Journal of the Operational Research Society 2003;54(3):255-262.

[9] A. S. Rababah et al. Novel hybrid method for interpolating missing information in body surface potential maps. In ISCE. 2019; .

[10] Stanley PC, Pilkington TC, Morrow MN. The Effects of Thoracic Inhomogeneities on the Relationship Between Epicardial and Torso Potentials. IEEE Transactions on Biomedical Engineering 1986;BME-33(3):273-284.

[11] Tikhonov AN, Arsenin VY. Solutions of Ill-posed Problems. Halsted Press, NY, 1977.

[12] Duchateau J, Potse M, Dubois R. Spatially coherent activation maps for electrocardiographic imaging. IEEE Trans Biomed Eng 2017;64(5):1149-1156.

Address for correspondence:

Dr. Y. Serinagaoglu Dogrusoz

Orta Dogu Teknik Universitesi

Elektrik and Elektronik Muh. Bol.

Ankara, Turkey

yserin@metu.edu.tr 